\title{
A DFT Study on Tautomer Stability of 4-Hydroxyquinoline Considering Solvent Effect and NBO Analysis
}

\author{
ZABIALAH HEIDARNEZHAD ${ }^{\mathrm{a},{ }^{*},}$, FATEMEH HEIDARNEZHAD ${ }^{\mathrm{a}}$, \\ IZATULLO GANIEV $^{\mathrm{b}}$, and ZIYODULLO OBIDOV ${ }^{\mathrm{b}}$ \\ ${ }^{\text {a }}$ Department of Chemistry, Islamic Azad University, Andimeshk , Iran \\ ${ }^{\mathrm{b}}$ Chemistry Institute, Tajikistan Academy of Sciences, Dushanbe, Tajikistan \\ z.heidarnezhad@gmail.com
}

Received 24 November 2012 / Accepted 15 December 2012

\begin{abstract}
Computational calculations at B3LYP/6-311++G (d,p) level were employed in the study of the predominant tautomeric of 4-hydroxyquinoline derivatives $\left(5-\mathrm{H}, 5-\mathrm{NO}_{2}, 5-\mathrm{Cl}, 5-\mathrm{OH}, 5-\mathrm{CH}_{3}\right.$, 6- $\mathrm{NO}_{2}, 6-\mathrm{Cl}, 6-\mathrm{OH}, 6-\mathrm{CH}_{3}, 7-\mathrm{NO}_{2}, 7-\mathrm{Cl}, 7-\mathrm{OH}, 7-\mathrm{CH}_{3}, 8-\mathrm{NO}_{2}, 8-\mathrm{Cl}, 8-\mathrm{OH}, 8-\mathrm{CH}_{3}$,) in the gas phase and selected solvents (benzene (non-polar solvent), tetrahydrofuran (THF) (polar aprotic solvent) and water (protic solvent)). The tautomers were also optimized in solvents according to the polarisable continuum method (PCM). For electron withdrawing and releasing derivatives the order of stability is $\mathrm{X} 1>\mathrm{X} 2$ $>\mathrm{X} 3$ and $\mathrm{X} 1$ form is a more stable and dominant form. An exception in the gas phase is $\mathrm{X} 2$ isomer of $(8-\mathrm{OH})$ which has two forms. In one, the hydrogen bond between hydroxyl substituted with nitrogen isn't formed, but in another it is formed. If the hydrogen bond is formed, X2 isomer is more stable than $\mathrm{X} 1$. In addition variation of dipole moments and charges on atoms in the solvents are studied.
\end{abstract}

Keywords: DFT study, NBO analysis, PCM model, 4-Hydroxyquinoline, Tautomerism

\section{Introduction}

The word quinoline in fact is derived from the word quinine, which in turn is derived from quina, a Spanish version of a local South American name for the bark of quinine-containing Cinchona species. The subsequent importance of quinoline is linked with malaria in the several successful antimalarial drugs such as chloroquine, which is also used in treatment of amoebic dysentery. Also the most general synthesis of these drugs has been through the 4-hydroxyquinoline. Apart from these, quinolines occur in plants as secondary metabolites (alkaloids) with quinine being the best known ${ }^{1}$. Among the bifunctional molecules, hydroxyquinolines have been widely studied from both experimental and theoretical viewpoints ${ }^{2}$. Early in 1968, Mason et al. ${ }^{3}$ had investigated the excited-state properties of 7- hydroxyquinolines (7-HQ) and pointed out that the $\mathrm{OH}$ group is more acidic and the ring nitrogen atom more basic in the excited state than in the ground state. 7-HQ has also been the subject of some theoretical investigations. The triple-proton-transfer reactions in the ground and first excited states of 7-HQ in methanol solution have been studied by using HF, MP2, CIS 
and CASSCF methods ${ }^{4}$. Tokay et al. studied tautomerism of 2-, 3- or 4-hydroxyquinoline derivatives along with their thio and azo analogs ${ }^{5}$. Shchavlev et al. studied rotation barriers, tautomerism, intramolecular hydrogen bond and solvent effects in 8-hydroxyquinoline by DFT computational ${ }^{6}$. Theoretical studies on proton transfer reactions of 8-hydroxyquinoline monomers and dimers have done and it implied that the hydrogen bond played an important role in depressing the activation energy of reaction ${ }^{7}$. In the present paper we studied tautomerism of 4hydroxyquinoline in the gas phase and solution using polarisable continuum method (PCM) at the B3LYP/6-311++G (d,p) level of theory.

\section{Experimental}

All these calculations were carried out on a corei7 computer by means of GAUSSIAN09 program package. First, all the compound's structures were drawn using Gauss View 03 and optimized in GAUSSIAN09. The tautomers were also optimized in solvents according to the polarisable continuum method of Tomasi and co-workers, which exploits the generating polyhedra procedure ${ }^{8-11}$ to build the cavity in the polarisable continuum medium, where the solute is accommodated. Atomic charges in all the structures were obtained using the Natural Population Analysis (NPA) method within the Natural Bond Orbital (NBO) approach ${ }^{12-14}$.

\section{Results and Discussion}

Structures and numbers of 4-hydroxyquinoline derivatives are depicted in Figure 1 and 2. The results of energy comparisons tautomers in the gas phase and different solvents are given in Table 1. In solvent phase X2 isomer is dominant. The order of stability in solvent phase is $\mathrm{X} 1>\mathrm{X} 2>\mathrm{X} 3 . \mathrm{X} 1$ and $\mathrm{X} 2$ isomers are both $\mathrm{N}$-amins, as it expected they are more stable than $\mathrm{X} 3$ isomers which is imine. In the gas phase $\mathrm{X} 1$ isomer is also dominant, with the exception of X2 isomer of (8-OH) form that is optimized to two forms which is depicted in Figure 2. At the first form, hydrogen bond between hydroxyl substituted $(\mathrm{OH})$ with nitrogen isn't formed then we consider it as X2 form.<smiles>Cc1ccc2c(c1)N=CCC2=O</smiles>

Figure 1. Tautomeric forms of 4-hydroxy quinoline.<smiles>COc1cccc2c(O)ccnc12</smiles><smiles>CCOc1cccc2c(O)ccnc12</smiles><smiles>Oc1ccnc2c(O)cccc12</smiles><smiles>Oc1ccnc2c(O)cccc12</smiles><smiles>[Y4][R4]([H])=C1CC=Nc2c(OC)cccc21</smiles>

$\mathrm{X} 3$ (input)<smiles>O=C1CC=Nc2c(OP)cccc21</smiles>

$\mathrm{x} 3$<smiles>[Y20][R10]=C1CC=Nc2c(O[Tl])cccc21</smiles>

$\mathrm{X} 3$ (input)

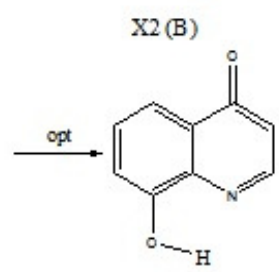

X3 (B)

Figure 2. Stationary Structure and optimized of $\mathrm{X} 1, \mathrm{X} 2, \mathrm{X} 3$ with $8-\mathrm{OH}$ situation 
Table 1. Total energies at B3LYP/6-311++G(d,p) in the gas phase and solvents

\begin{tabular}{|c|c|c|c|c|c|}
\hline C-R & Tautomer & $\operatorname{Gas}(1.0)$ & Benzene(2.2) & THF(7.9) & Water(78.54) \\
\hline \multirow[t]{3}{*}{$5-\mathrm{H}$} & $\mathrm{X} 1$ & -477.2877453 & -477.2961726 & -477.3021367 & -477.3049159 \\
\hline & $\mathrm{X} 2$ & -477.2816309 & -477.2870899 & -477.290546 & -477.292516 \\
\hline & $\mathrm{X} 3$ & -477.265782 & -477.2712432 & -477.274696 & -477.2761871 \\
\hline \multirow[t]{3}{*}{$5-\mathrm{NO}_{2}$} & $\mathrm{X} 1$ & -681.8341753 & -681.8467459 & -681.8557466 & -681.859961 \\
\hline & $\mathrm{X} 2$ & -681.8304161 & -681.8386952 & -681.8438853 & -681.8460108 \\
\hline & $\mathrm{X} 3$ & -681.8131533 & -681.8218036 & -681.8274844 & -6818298817 \\
\hline \multirow[t]{3}{*}{$5-\mathrm{Cl}$} & $\mathrm{X} 1$ & -936.8982053 & -936.9072757 & -936.9138317 & -936.9168042 \\
\hline & $\mathrm{X} 2$ & -936.8937659 & -936.8993989 & -936.9029489 & -936.9043978 \\
\hline & X3 & -936.8778688 & -936.8838108 & -936.8876389 & -936.889226 \\
\hline \multirow[t]{3}{*}{$5-\mathrm{CH}_{3}$} & $\mathrm{X} 1$ & -516.6115067 & -516.6189948 & -516.6242409 & -516.6266708 \\
\hline & $\mathrm{X} 2$ & -516.6037536 & -516.6089159 & -516.6122544 & -516.613722 \\
\hline & $\mathrm{X} 3$ & -516.5903093 & -516.5952858 & -516.5984212 & -516.5997726 \\
\hline \multirow[t]{3}{*}{$5-\mathrm{OH}$} & $\mathrm{X} 1$ & -552.5498916 & -552.5578293 & -552.5633784 & -552.5658462 \\
\hline & $\mathrm{X} 2$ & -552.5302909 & -552.5369148 & -552.5412133 & -552.5430067 \\
\hline & X3 & -552.5258144 & -552.5312048 & -552.5345845 & -552.535965 \\
\hline \multirow[t]{3}{*}{$6-\mathrm{NO}_{2}$} & $\mathrm{X} 1$ & -681.849861 & -681.8612292 & -681.8692131 & -681.8729173 \\
\hline & $\mathrm{X} 2$ & -681.8444538 & -681.8523581 & -681.8573956 & -681.8594943 \\
\hline & $\mathrm{X} 3$ & -681.8260318 & -681.8341119 & -681.8392801 & -681.8414311 \\
\hline \multirow[t]{3}{*}{$6-\mathrm{Cl}$} & $\mathrm{X} 1$ & -936.9090792 & -936.917665 & -936.9237708 & -936.9265152 \\
\hline & $\mathrm{X} 2$ & -936.9039818 & -936.909358 & -936.9127584 & -936.914154 \\
\hline & $\mathrm{X} 3$ & -936.8871807 & -936.8926042 & -936.8960195 & -936.8974222 \\
\hline \multirow[t]{3}{*}{$6-\mathrm{CH}_{3}$} & $\mathrm{X} 1$ & -516.6149904 & -516.6234023 & -516.6293534 & -516.6321269 \\
\hline & $\mathrm{X} 2$ & -516.6088008 & -516.6142604 & -516.61776 & -516.6192888 \\
\hline & $\mathrm{X} 3$ & -516.5936713 & -516.5991912 & -516.6026813 & -516.6041887 \\
\hline \multirow[t]{3}{*}{ C6-OH } & $\mathrm{X} 1$ & -552.5350491 & -552.5452791 & -552.5521995 & -552.5552048 \\
\hline & $\mathrm{X} 2$ & -552.5295309 & -552.5368536 & -552.5414488 & -552.543323 \\
\hline & $\mathrm{X} 3$ & -552.5137838 & -552.5210603 & -552.5255349 & -552.527338 \\
\hline \multirow[t]{3}{*}{ 7- $\mathrm{NO}_{2}$} & $\mathrm{X} 1$ & -681.8485593 & -681.8589573 & -681.8660425 & -681.8691386 \\
\hline & $\mathrm{X} 2$ & -681.8433271 & 11364 & -681.8561379 & -681.858232 \\
\hline & X3 & -681.8248417 & -681.8325341 & -681.8373812 & -681.8393728 \\
\hline \multirow[t]{3}{*}{ 7-Cl } & $\mathrm{X} 1$ & -936.9097793 & -936.9181033 & -936.9239211 & -936.9265048 \\
\hline & $\mathrm{X} 2$ & -936.9043448 & -936.9097021 & -936.9131172 & -936.9145326 \\
\hline & $\mathrm{X} 3$ & -936.8879896 & -936.8933757 & -936.8967526 & -936.8981348 \\
\hline \multirow[t]{3}{*}{ 7- $\mathrm{CH}_{3}$} & $\mathrm{X} 1$ & -516.6156274 & -516.6241515 & -516.6302146 & -516.6330483 \\
\hline & $\mathrm{X} 2$ & -516.610003 & -516.6154843 & -516.61776 & -516.6205267 \\
\hline & $\mathrm{X} 3$ & -516.5944621 & -516.6000944 & -516.6036699 & -516.6052182 \\
\hline \multirow[t]{3}{*}{$7-\mathrm{OH}$} & $\mathrm{X} 1$ & -552.5366461 & -552.5470699 & -552.554143 & -552.5572149 \\
\hline & $\mathrm{X} 2$ & -552.5308576 & -552.5381364 & -552.5426477 & -552.5444694 \\
\hline & $\mathrm{X} 3$ & -552.5159576 & -552.523576 & -552.5283221 & -552.5302579 \\
\hline \multirow[t]{3}{*}{$8-\mathrm{NO}_{2}$} & $\mathrm{X} 1$ & -681.8524071 & -681.8604231 & -681.8657324 & -681.8679972 \\
\hline & $\mathrm{X} 2$ & -681.8336558 & 26087 & -681.8484754 & -681.8509664 \\
\hline & $\mathrm{X} 3$ & -681.8159934 & -681.8246576 & -681.8301925 & -681.8324876 \\
\hline \multirow[t]{3}{*}{$8-\mathrm{Cl}$} & $\mathrm{X} 1$ & -936.9102968 & -936.91728 & -936.9221247 & -936.9242687 \\
\hline & $\mathrm{X} 2$ & -936.9003076 & -936.9064727 & -936.9104637 & -936.9121332 \\
\hline & X3 & -936.8832317 & -936.8892072 & -936.8930222 & -936.8945926 \\
\hline \multirow[t]{3}{*}{$8-\mathrm{CH}_{3}$} & $\mathrm{X} 1$ & -516.6150235 & -516.6233332 & -516.6292595 & -516.6320312 \\
\hline & $\mathrm{X} 2$ & -516.6098154 & -516.6147717 & -516.6179225 & -516.6192913 \\
\hline & X3 & -516.5931177 & -516.5982474 & -516.6015069 & -516.6029196 \\
\hline \multirow[t]{5}{*}{$8-\mathrm{OH}$} & $\mathrm{X} 1$ & -552.5362293 & -552.5455198 & -552.5519222 & -552.5547455 \\
\hline & X2 & -552.5239723 & -552.5325723 & -552.5380869 & -552.5403496 \\
\hline & X2(B) & -552.5376213 & -552.5430497 & -552.5464555 & -552.5478522 \\
\hline & X3 & -552.5074838 & -552.5160866 & -552.5215156 & -552.5237662 \\
\hline & $\mathrm{X} 3(\mathrm{~B})$ & -552.5202238 & -552.5258915 & -552.5294623 & -552.5309337 \\
\hline
\end{tabular}


The energy stability is $\mathrm{X} 1>\mathrm{X} 2>\mathrm{X} 3$. At second form, the hydrogen bond between them is formed. In this case X2(B) isomer with substituted energy between X1, X2(B) with $0.878 \mathrm{kcal} \mathrm{mol}^{-1}$ is more dominant than $\mathrm{X} 1$. Then the stability order is $\mathrm{X} 2(\mathrm{~B})>\mathrm{X} 1>\mathrm{X} 3$. It is to mention that because of the solvent effect on the hydrogen bond only $\mathrm{X} 2(\mathrm{~B})$ is more predominant than $\mathrm{X} 1$ in gas phase. Although, the hydrogen bond of X2(B) causes more stability and negative energy than $\mathrm{X} 2$ (A) in solvent phase, but this change is not that much to stabilize $\mathrm{X} 2$ (B) form than X1 in solvent phase. Definitely in X3 form the hydrogen bond is formed, but given the fact that there is a large difference in energy stability of X3 with X1, X2. The hydrogen bond effect also can not compensate this difference and no change happens in the stability of $\mathrm{X} 3$ form compared to $\mathrm{X} 1$ and $\mathrm{X} 2$. The difference of (E2-E1) between $\mathrm{X} 2$ and $\mathrm{X} 1$ forms in gas phase is belonged to derivates with this subsisted situation $(5-\mathrm{OH}),\left(6-\mathrm{CH}_{3}\right),(7-\mathrm{OH}),\left(8-\mathrm{NO}_{2}\right)$ and their energy is $12.299,3.884,3.630,11.766$ respectively on the basis of $\left(\mathrm{kcal} \mathrm{mol}^{-1}\right)$. Solvent effects are relevant to the tautomers stability phenomena, since polarity differences among the tautomers can induce significant changes in their relative energies in solution, PCM/B3LYP calculations were used to analyze the solvent effects on tautomerism of 4-hydroxyquinoline. It is important to stress that the PCM model does not consider the presence of explicit solvent molecules; hence specific solute-solvent interactions are not described and the calculated solvation effects arises only from mutual solute-solvent electrostatic polarization. Calculated dipole moments for the Xs are presented in Table 2. The solvent represented by a polarizable continuum is found to show significant effect on the dipole moments of the individual solute conformers. The dipole moments increase by changing the gas phase to the solution. For all conforms, the most obtained dipole moment is seen in water solvent and gas phase. The biggest difference of dipole moment of all conforms belongs to $\mathrm{X} 1$ with substituent situation $\left(5-\mathrm{NO}_{2}\right)$ is $14.3963 \mathrm{D}, 4.3972 \mathrm{D}$ for water solution and gas phase respectively. For all conforms with substituent situation (5-R), (6-R) the most dipole moment belongs to $\mathrm{X} 1$ with $\mathrm{R}=\mathrm{NO}_{2}$, in (7-R) it belongs to $\mathrm{X} 2$ with $\mathrm{R}=\mathrm{NO}_{2}$, but in (8-R) it belongs to $\mathrm{X} 2$ with $\mathrm{R}=\mathrm{OH}$. We have examined the charge distribution of tautomers in the solvent as well as gas phase by using calculated NBO charges (Table 3,4 \& 5). The decrease of negative charge was observed on (N) atom in $\mathrm{X} 1$ form when the phase is changed from gas phase to polar solvent. With the exception of the $\mathrm{X} 1$ with substituent situation $(8-\mathrm{OH})$ and all $\mathrm{X} 2$ and $\mathrm{X} 3$ isomers, the phase is changed from gas to polar solvent the negative charge on $\mathrm{N}$ increases.

Table 2. Calculated dipole moments in the gas phase and solvents

\begin{tabular}{cccccc}
\hline C-R & Tautomer & Gas & Benzene & THF & Water \\
\hline $5-\mathrm{H}$ & $\mathrm{X} 1$ & 6.2321 & 7.6598 & 8.7957 & 9.3584 \\
& $\mathrm{X} 2$ & 2.6005 & 3.1475 & 3.5613 & 3.7602 \\
& $\mathrm{X} 3$ & 1.451 & 1.6685 & 1.8307 & 1.9075 \\
$5-\mathrm{NO}_{2}$ & $\mathrm{X} 1$ & 9.9991 & 12.0424 & 13.6239 & 14.3963 \\
& $\mathrm{X} 2$ & 2.9567 & 3.2832 & 3.509 & 3.6143 \\
& $\mathrm{X} 3$ & 5.31 & 6.169 & 6.7924 & 7.0771 \\
$5-\mathrm{Cl}$ & $\mathrm{X} 1$ & 7.3173 & 9.0429 & 10.417 & 11.0766 \\
& $\mathrm{X} 2$ & 1.7747 & 2.0978 & 2.3356 & 2.4437 \\
& $\mathrm{X} 3$ & 2.6787 & 3.2056 & 3.6016 & 3.7854 \\
$5-\mathrm{CH}_{3}$ & $\mathrm{X} 1$ & 5.6115 & 6.9293 & 7.9792 & 8.4993 \\
& $\mathrm{X} 2$ & 2.8697 & 3.5039 & 3.9931 & 4.2309 \\
& $\mathrm{X} 3$ & 0.9581 & 1.0932 & 1.1929 & 1.2409 \\
$5-\mathrm{OH}$ & $\mathrm{X} 1$ & 6.6742 & 8.1693 & 9.3536 & 9.9196 \\
& $\mathrm{X} 2$ & 3.506 & 4.258 & 4.827 & 5.089 \\
\hline & & & & & Contd...
\end{tabular}




\begin{tabular}{|c|c|c|c|c|c|}
\hline & X3 & 1.9678 & 2.3459 & 2.6319 & 2.7657 \\
\hline \multirow[t]{3}{*}{$6-\mathrm{NO}_{2}$} & $\mathrm{X} 1$ & 9.5081 & 11.3729 & 12.8312 & 13.5491 \\
\hline & $\mathrm{X} 2$ & 6.2037 & 7.1799 & 7.9096 & 8.2528 \\
\hline & X3 & 5.886 & 6.8419 & 7.5547 & 7.8872 \\
\hline \multirow{3}{*}{ 6-Cl } & $\mathrm{X} 1$ & 7.1479 & 8.7072 & 9.934 & 10.517 \\
\hline & $\mathrm{X} 2$ & 3.2454 & 3.828 & 4.2547 & 4.4489 \\
\hline & $\mathrm{X} 3$ & 2.6072 & 3.0687 & 3.4035 & 3.5559 \\
\hline \multirow[t]{3}{*}{$6-\mathrm{CH}_{3}$} & $\mathrm{X} 1$ & 6.0299 & 7.4474 & 8.5841 & 9.151 \\
\hline & $\mathrm{X} 2$ & 2.6301 & 3.1865 & 3.6112 & 3.8168 \\
\hline & $\mathrm{X} 3$ & 1.3757 & 1.55 & 1.6804 & 1.7431 \\
\hline \multirow[t]{3}{*}{ 6-OH } & $\mathrm{X} 1$ & 5.2436 & 6.513 & 7.5306 & 8.0205 \\
\hline & $\mathrm{X} 2$ & 3.6682 & 4.3917 & 4.9364 & 5.186 \\
\hline & $\mathrm{X} 3$ & 0.3808 & 0.4894 & 0.5724 & 0.61 \\
\hline \multirow[t]{3}{*}{ 7- $\mathrm{NO}_{2}$} & $\mathrm{X} 1$ & 5.54 & 6.6605 & 7.5598 & 7.9939 \\
\hline & $\mathrm{X} 2$ & 7.6709 & 8.8954 & 9.8018 & 10.2241 \\
\hline & X3 & 4.5309 & 5.2187 & 5.7194 & 5.9471 \\
\hline \multirow[t]{3}{*}{ 7-Cl } & $\mathrm{X} 1$ & 5.4069 & 6.6817 & 7.7001 & 8.1879 \\
\hline & $\mathrm{X} 2$ & 4.3562 & 5.1484 & 5.7335 & 6.0025 \\
\hline & X3 & 1.4499 & 1.7371 & 1.9431 & 2.0356 \\
\hline \multirow[t]{3}{*}{ 7- $\mathrm{CH}_{3}$} & $\mathrm{X} 1$ & 6.5437 & 8.0462 & 9.2412 & 9.8338 \\
\hline & $\mathrm{X} 2$ & 2.2407 & 2.7344 & 3.1175 & 3.3018 \\
\hline & X3 & 2.0031 & 2.2998 & 2.5184 & 2.6221 \\
\hline \multirow[t]{3}{*}{ 7-OH } & $\mathrm{X} 1$ & 5.1298 & 6.3782 & 7.3825 & 7.8667 \\
\hline & $\mathrm{X} 2$ & 2.0543 & 2.473 & 2.7897 & 2.9358 \\
\hline & X3 & 2.8032 & 3.2931 & 3.6557 & 3.8228 \\
\hline \multirow{3}{*}{$8-\mathrm{NO}_{2}$} & $\mathrm{X} 1$ & 0.896 & 1.3667 & 1.7776 & 1.9846 \\
\hline & $\mathrm{X} 2$ & 7.1056 & 8.3267 & 9.259 & 9.7053 \\
\hline & X3 & 3.0084 & 3.4425 & 3.7557 & 3.9026 \\
\hline \multirow[t]{3}{*}{$8-\mathrm{Cl}$} & $\mathrm{X} 1$ & 4.4466 & 5.5925 & 6.522 & 6.9721 \\
\hline & $\mathrm{X} 2$ & 4.2436 & 5.0948 & 5.7366 & 6.0335 \\
\hline & X3 & 0.2256 & 0.3447 & 0.4328 & 0.4721 \\
\hline \multirow[t]{3}{*}{$8-\mathrm{CH}_{3}$} & $\mathrm{X} 1$ & 6.6548 & 8.1798 & 9.3817 & 9.9748 \\
\hline & $\mathrm{X} 2$ & 2.2092 & 2.6537 & 2.9851 & 3.143 \\
\hline & X3 & 2.0008 & 2.3178 & 2.553 & 2.6647 \\
\hline \multirow[t]{5}{*}{$8-\mathrm{OH}$} & $\mathrm{X} 1$ & 6.6527 & 8.1272 & 9.3013 & 9.8676 \\
\hline & X2 & 2.1459 & 2.6713 & 3.0909 & 3.22906 \\
\hline & $\mathrm{X} 2(\mathrm{~B})$ & 3.8466 & 4.5561 & 5.0822 & 5.3247 \\
\hline & X3 & 2.224 & 2.5142 & 2.7196 & 2.8136 \\
\hline & X3(B) & 1.7186 & 2.0908 & 2.3685 & 2.4952 \\
\hline
\end{tabular}

Table 3. Calculated NBO charge for $5-\mathrm{H}$ and $5-\mathrm{R}$ positions

\begin{tabular}{|c|c|c|c|c|c|c|c|c|c|c|c|c|c|}
\hline & $\mathrm{e}=$ & 1.0 & 2.2 & 7.6 & 78.4 & 1.0 & 2.2 & 7.6 & 78.4 & 1.0 & 2.2 & 7.6 & 78.4 \\
\hline C-R & Atom & $\mathrm{X}_{1}$ & & & & $\mathrm{X}_{2}$ & & & & $\mathrm{X}_{3}$ & & & \\
\hline \multirow[t]{6}{*}{$5-\mathrm{H}$} & N1 & -0.541 & -0.536 & -0.529 & -0.525 & -0.468 & -0.497 & -0.519 & -0.529 & -0.458 & -0.48 & -0.495 & -0.502 \\
\hline & $\mathrm{C} 2$ & 0.0637 & 0.0746 & 0.085 & 0.088 & 0.087 & 0.0836 & 0.0813 & 0.08 & 0.174 & 0.183 & 0.189 & 0.192 \\
\hline & C3 & -0.332 & -0.348 & -0.359 & -0.366 & -0.34 & -0.342 & -0.342 & -0.342 & -0.556 & -0.56 & -0.566 & -0.567 \\
\hline & $\mathrm{C} 4$ & 0.472 & 0.4713 & 0.469 & 0.471 & 0.375 & 0.3797 & 0.383 & 0.385 & 0.556 & 0.568 & 0.575 & 0.58 \\
\hline & C5 & -0.142 & -0.152 & -0.161 & -0.166 & -0.174 & -0.18 & -0.184 & -0.185 & -0.139 & -0.14 & -0.145 & -0.147 \\
\hline & C6 & -0.216 & -0.222 & -0.225 & -0.225 & -0.2 & -0.206 & -0.21 & -0.212 & -0.201 & -0.21 & -0.209 & -0.21 \\
\hline
\end{tabular}




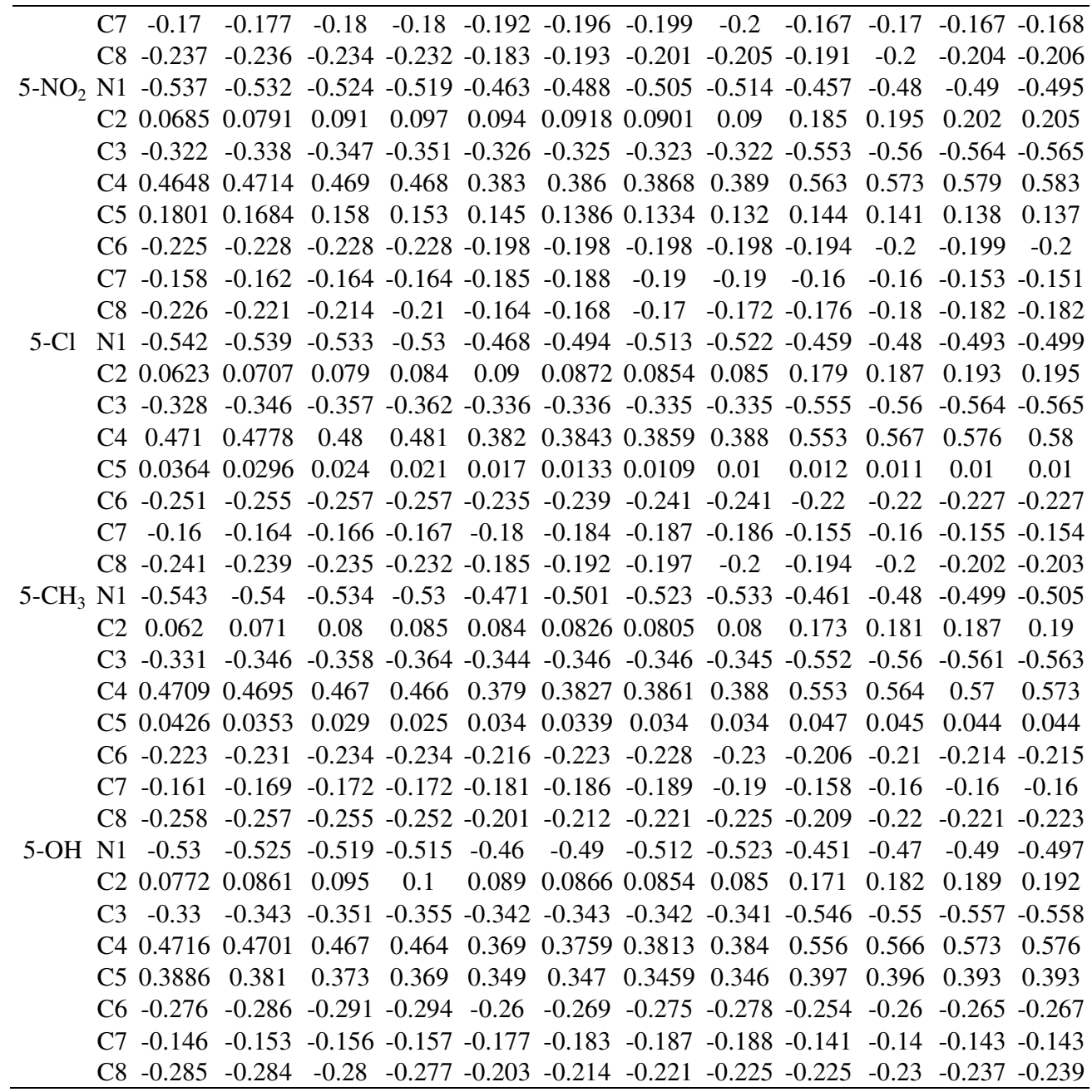

Table 4. Calculated BO charge for $6-\mathrm{R}$ and $7-\mathrm{R}$ positions

\begin{tabular}{|c|c|c|c|c|c|c|c|c|c|c|c|c|c|}
\hline \multirow[b]{2}{*}{ C-R } & $\mathrm{e}=$ & 1.0 & 2.2 & 7.6 & 78.4 & 1.0 & 2.2 & 7.6 & 78.4 & 1.0 & 2.2 & 7.6 & 78.4 \\
\hline & Atom & $\mathrm{X} 1$ & & & & $\mathrm{X} 2$ & & & & $\mathrm{X} 3$ & & & \\
\hline \multirow[t]{8}{*}{ 6- $\mathrm{NO}_{2}$} & N1 & -0.54 & -0.53 & -0.523 & -0.518 & -0.47 & -0.48 & -0.51 & -0.52 & -0.46 & -0.482 & -0.5 & -0.501 \\
\hline & $\mathrm{C} 2$ & 0.065 & 077 & 0.089 & 0.095 & 0.103 & 0.092 & 0.103 & 0.103 & 0.193 & 0.205 & 0.214 & 0.218 \\
\hline & C3 & -0.32 & -0.33 & -0.342 & -0.346 & -0.34 & -0.32 & -0.33 & -0.33 & -0.56 & -0.566 & -0.57 & -0.572 \\
\hline & $\mathrm{C} 4$ & 0.473 & 0.477 & 0.477 & 0.477 & 0.388 & 0.381 & 0.399 & 0.402 & 0.558 & 0.572 & 0.581 & 0.585 \\
\hline & C5 & -0.12 & -0.12 & -0.122 & -0.124 & -0.15 & -0.16 & -0.14 & -0.14 & -0.13 & -0.123 & -0.12 & -0.12 \\
\hline & C6 & 0.056 & 0.055 & 0.054 & 0.054 & 0.072 & -0.2 & 0.068 & 0.067 & 0.069 & 0.069 & 0.067 & 0.067 \\
\hline & C7 & -0.16 & -0.16 & -0.164 & -0.165 & -0.19 & 0.068 & -0.19 & -0.19 & -0.15 & -0.151 & -0.15 & -0.147 \\
\hline & C8 & -0.22 & -0.22 & -0.215 & -0.212 & -0.17 & -0.14 & -0.18 & -0.18 & -0.18 & -0.187 & -0.19 & -0.19 \\
\hline \multirow[t]{3}{*}{$6-\mathrm{Cl}$} & N1 & -0.54 & -0.54 & -0.531 & -0.528 & -0.46 & -0.49 & -0.51 & -0.52 & -0.46 & -0.478 & -0.49 & -0.499 \\
\hline & $\mathrm{C} 2$ & 0.067 & 0.075 & 0.083 & 0.088 & 0.087 & 0.085 & 0.084 & 0.084 & 0.176 & 0.185 & 0.192 & 0.195 \\
\hline & C3 & -0.33 & -0.35 & -0.36 & -0.364 & -0.33 & -0.34 & -0.33 & -0.33 & -0.56 & -0.563 & -0.57 & -0.568 \\
\hline
\end{tabular}




\begin{tabular}{|c|c|c|c|c|c|c|c|c|c|c|c|c|c|}
\hline & & 471 & 477 & & & $0.3 / 6$ & $0.3 / 9$ & 0.382 & 0.383 & 0.538 & $0.5 / 1$ & & \\
\hline & C5 & -0.17 & -0.18 & & & -0.2 & -0.21 & -0.21 & -0.21 & -0.17 & .169 & .17 & 0.172 \\
\hline & C6 & -0.05 & -0.05 & & & -0.03 & -0.04 & -0.04 & -0.04 & -0.04 & -0.043 & .04 & \\
\hline & C7 & -0.19 & -0.2 & & & -0.21 & -0.22 & -0.22 & -0.22 & 0.19 & & & \\
\hline & C8 & & & & & & & & & & & & \\
\hline \multirow[t]{8}{*}{$6-\mathrm{CH}_{3}$} & N1 & -0.54 & -0.54 & & -0.524 & -0.47 & -0.5 & -0.52 & -0.53 & -0.46 & -0.479 & -0.49 & -0.5 \\
\hline & $\mathrm{C} 2$ & 0.067 & 0.075 & & & 0.083 & 0.08 & 0.078 & & 169 & & 183 & \\
\hline & C3 & & & & & -0.34 & -0.34 & & & & & -0.56 & \\
\hline & C4 & & & & & 0.374 & 0.377 & & & & & & \\
\hline & C5 & .14 & -0.15 & & & -0.18 & -0.18 & -0.19 & & & & & \\
\hline & C6 & & & & & -0.03 & & & & 0.03 & & .03 & \\
\hline & & & & & & -0.19 & & & & & & 0.17 & \\
\hline & & & & & & -0.18 & & & & & & & \\
\hline \multirow[t]{8}{*}{ 6-OH } & N1 & -0.54 & -0.53 & & & -0.46 & -0.49 & -0.51 & -0.52 & .46 & & 0.49 & \\
\hline & $\mathrm{C} 2$ & 0.065 & & & & 0.075 & 0.071 & & & & & & \\
\hline & C & & & & & -0.33 & & & & & & & \\
\hline & & & 0.466 & & & 0.364 & 0.368 & & & & & & \\
\hline & & -0.23 & -0.24 & & & -0.27 & -0.27 & -0 . & & & & & \\
\hline & & & & & & 0.321 & & & & & & & \\
\hline & & & & & & -0.23 & & & & & & & \\
\hline & & -0.21 & -0.21 & & & -0.16 & -0.17 & -0.18 & -0.19 & & & 18 & 0.187 \\
\hline \multirow[t]{8}{*}{ 7- $-\mathrm{NO}_{2}$} & IVI & -0.54 & -0.53 & & & -0.46 & -0.48 & & & & & & \\
\hline & $\mathrm{C} 2$ & & & & & 0.094 & & & & & & & \\
\hline & & & & & & -0.32 & & & & & & & \\
\hline & & & & & & 0.377 & & & & & & & \\
\hline & & & & & & -0.16 & & & & & & & \\
\hline & & & & & & & & & & & & & \\
\hline & & 0 & 0.0 & & & 0.068 & 0.0 & 0.0 & & & & & \\
\hline & C8 & & & & & -0.14 & & & & & & & -0.16 \\
\hline \multirow[t]{8}{*}{ 7-Cl } & N1 & & & & & -0.47 & & & & & & & \\
\hline & & & & & & 0.093 & & & & & & & \\
\hline & & & & & & -0.34 & 4 & & & & & & \\
\hline & C & & & & & 0.378 & & & & & & & \\
\hline & & & & & & -0.16 & & & & & & & \\
\hline & & & & & & & & & & & & & \\
\hline & & & & & & -0.04 & .04 & & & & & & \\
\hline & & & & & & -0.19 & & & & & & & 228 \\
\hline \multirow[t]{8}{*}{ 7- $-\mathrm{CH}_{3}$} & N1 & & & & & & & & & & & & \\
\hline & & & & & & & & & & & & & \\
\hline & & & & & & & & & & & & & \\
\hline & C4 & & & & & 0.379 & & & & & & & \\
\hline & & & & & & & & & & & & & \\
\hline & & & & & & & & & & & & & \\
\hline & & & & & & & & & & -0 & & & \\
\hline & C8 & -0.22 & -0.22 & & & -0.17 & -0.18 & -0.19 & -0.2 & -0.18 & & -0.19 & -0.191 \\
\hline \multirow[t]{8}{*}{ 7-OH } & N1 & -0.54 & -0.54 & -0.5 & -0.528 & -0.48 & -0.51 & -0.53 & -0.54 & -0.46 & -0.484 & -0.5 & -0.504 \\
\hline & $\mathrm{C} 2$ & 0.063 & 0.073 & 0.0 & 0.087 & 0.091 & 0.087 & 0.084 & 0.082 & 0.181 & 0.188 & 0.194 & 0.196 \\
\hline & C3 & -0.33 & -0.35 & -0.3 & -0.366 & -0.35 & -0.35 & -0.35 & -0.35 & -0.55 & -0.56 & -0.56 & -0.565 \\
\hline & C4 & 0.472 & 0.472 & & 0.4 & 0.38 & 0.384 & 0.387 & 0.388 & 0.551 & 0.561 & 0.568 & 0.571 \\
\hline & C5 & -0.12 & -0.13 & -0.14 & -0.145 & -0.15 & -0.16 & -0.16 & -0.17 & -0.12 & -0.123 & -0.13 & -0.128 \\
\hline & $\mathrm{Ce}$ & -0.29 & -0.29 & -0.2 & & -0.23 & -0.24 & -0.25 & -0.25 & -0.25 & -0.254 & -0.26 & -0.26 \\
\hline & $c$ & 0.343 & 0.342 & 0.3 & 0.342 & 0.328 & 0.327 & 0.327 & 0.327 & 0.348 & 0.352 & 0.355 & 0.357 \\
\hline & C & -0.29 & -0.3 & -0.29 & -0.297 & -0.28 & -0.29 & -0.29 & -0.29 & -0.28 & -0.279 & -0.28 & -0.281 \\
\hline
\end{tabular}


Table 5. Calculated NBO charge for 8-R position

\begin{tabular}{|c|c|c|c|c|c|c|c|c|c|c|c|c|c|}
\hline & $e=$ & 1.0 & 2.2 & 7.6 & 78.4 & 1.0 & 2.2 & 7.6 & 78.4 & 1.0 & 2.2 & 7.6 & 78.4 \\
\hline C-R & Atom & $\mathrm{X} 1$ & & & & $\mathrm{X} 2$ & & & & $\mathrm{X} 3$ & & & \\
\hline \multirow{8}{*}{$8-\mathrm{NO}_{2}$} & $\mathrm{~N} 1$ & -0.541 & & -0.524 & -0.52 & -0.457 & & -0.504 & -0.516 & -0.452 & & & \\
\hline & $\mathrm{C} 2$ & & & 0.09 & 0.094 & 0.103 & 0.1 & & 0.098 & 0.195 & & & -0 \\
\hline & C3 & & & 336 & -0.34 & -0.331 & -0.333 & .331 & -0.33 & .559 & & & -0.57 \\
\hline & C4 & & & 477 & 0.477 & 0.381 & 0.387 & 0.3918 & 0.395 & 0.561 & $5 / 33$ & 58 & $.58<$ \\
\hline & C5 & -0.099 & & -0.099 & -0.1 & -0.155 & & 149 & -0.145 & -0.128 & 126 & 0.12 & $-0.12 ?$ \\
\hline & C6 & -0.217 & & -0.219 & -0.22 & -0.194 & 199 & -0.203 & -0.205 & -0.19 & -0.192 & -0.19 & -0.193 \\
\hline & C7 & & & -0 . & -0.15 & -0.176 & & -0.171 & -0.17 & -0.162 & -0.16 & -0.16 & -0.157 \\
\hline & C8 & 3 & 0.067 & 0.071 & 0.073 & 0.103 & 0.092 & 0.0838 & 0.08 & 0.097 & 0.089 & 0.083 & 0.082 \\
\hline \multirow[t]{8}{*}{$8-\mathrm{Cl}$} & N1 & -0 & & -0.533 & -0.53 & -0.457 & -0.485 & -0.505 & -0.516 & -0.449 & -0.471 & -0.49 & -0.493 \\
\hline & $\mathrm{C} 2$ & 0.06 & & 086 & 0.089 & 0.094 & 0.092 & 0.0898 & 0.089 & 0.184 & 0.1932 & 0.2 & 0.202 \\
\hline & C3 & & & & -0.36 & -0.335 & & & .334 & -0.558 & & -0.57 & -0.56 \\
\hline & $\mathrm{C} 4$ & 0.47 & & 0.481 & 0.482 & 0.377 & 0.38 & 0.38 & 0.386 & 0.559 & & 0.58 & 0.584 \\
\hline & C5 & -0.1 & & & -0.16 & -0.178 & & & -0.181 & -0.147 & & -0.15 & -0.14 \\
\hline & C6 & & & & -0.21 & -0.188 & & & & & & -0.19 & -0.19 \\
\hline & C7 & -0.196 & & & -0.2 & -0.212 & -0.2 & -0.217 & -0.217 & -0.189 & & -0.19 & -0.18 \\
\hline & C & & & & -0.06 & -0.028 & & -0.04 & & -0.035 & & -0.04 & -0.04 \\
\hline \multirow[t]{8}{*}{$8-\mathrm{CH}_{3}$} & N1 & -0.54 & -0.5 & -0.528 & -0.52 & -0.476 & -0.496 & -0.512 & -0.521 & -0.463 & -0.479 & -0.49 & -0.49 \\
\hline & $\mathrm{C} 2$ & 0.0 & & & 0.09 & 0.089 & 0.084 & 0.0807 & 0.08 & 0.178 & & 0.19 & 0.193 \\
\hline & C3 & -0.329 & & -0.355 & -0.36 & -0.341 & -0.342 & -0.342 & -0.341 & -0.556 & -0.562 & -0.57 & $-0.56^{\prime}$ \\
\hline & C4 & 0.467 & & & 0.469 & 0.377 & & 0.382 & 0.384 & 0.555 & 0.5684 & 0.576 & 0.578 \\
\hline & C5 & -0.148 & -0.159 & -0.168 & -0.17 & -0.184 & -0.19 & -0.194 & -0.196 & -0.148 & -0.153 & -0.16 & -0.157 \\
\hline & C6 & & & & -0.22 & -0.192 & -0.198 & -0.203 & -0.2 & -0.193 & -0.199 & -0.2 & -0.20 \\
\hline & & & & & -0.19 & -0.206 & & & & -0.18 & -0.1 & -0.18 & -0.18 \\
\hline & C8 & $\begin{array}{c}-0.033 \\
\mathrm{X} 1\end{array}$ & -0.028 & -0.023 & -0.02 & $\begin{array}{c}0.02 \\
\mathrm{X} 2\end{array}$ & 0.015 & 0.0106 & 0.008 & $\begin{array}{c}0.012 \\
\mathrm{X} 2(\mathrm{~B})\end{array}$ & 0.0092 & $0.00^{\circ}$ & 0.005 \\
\hline
\end{tabular}

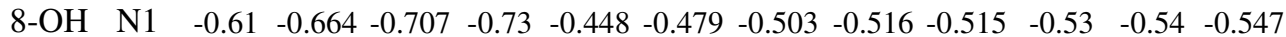

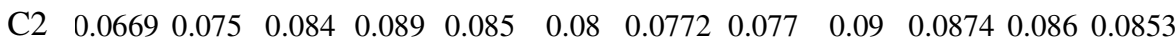

$\begin{array}{lllllllllllll}\text { C3 } & -0.334 & -0.348 & -0.358 & -0.36 & -0.335 & -0.338 & -0.338 & -0.339 & -0.34 & -0.34 & -0.34 & -0.337\end{array}$

$\begin{array}{lllllllllllll}\mathrm{C} 4 & 0.4692 & 0.471 & 0.468 & 0.467 & 0.373 & 0.377 & 0.3805 & 0.384 & 0.382 & 0.3848 & 0.387 & 0.3881\end{array}$

$\begin{array}{llllllllllllll}\text { C5 } & -0.174 & -0.185 & -0.196 & -0.2 & -0.209 & -0.217 & -0.221 & -0.222 & -0.216 & -0.221 & -0.22 & -0.225\end{array}$

$\begin{array}{lllllllllllll}\text { C6 } & -0.197 & -0.202 & -0.203 & -0.2 & -0.181 & -0.187 & -0.19 & -0.191 & -0.173 & -0.181 & -0.19 & -0.189\end{array}$

$\begin{array}{lllllllllllll}\text { C7 } & -0.271 & -0.273 & -0.273 & -0.27 & -0.296 & -0.297 & -0.297 & -0.296 & -0.255 & -0.263 & -0.27 & -0.271\end{array}$

$\begin{array}{llllllllllllll}\mathrm{C} 8 & 0.3171 & 0.32 & 0.325 & 0.327 & 0.358 & 0.353 & 0.3479 & 0.345 & 0.334 & 0.3279 & 0.323 & 0.3211\end{array}$ $\mathrm{X} 3 \quad \mathrm{X} 3(\mathrm{~B})$

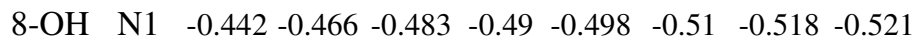

$\begin{array}{llllllllll}\mathrm{C} 2 & 0.1719 & 0.179 & 0.184 & 0.187 & 0.181 & 0.19 & 0.1966 & 0.199\end{array}$

C3 $-0.557-0.562-0.566-0.57 \quad-0.556-0.561 \quad-0.565-0.567$

$\begin{array}{lllllllll}\mathrm{C} 4 & 0.5584 & 0.57 & 0.577 & 0.58 & 0.557 & 0.569 & 0.5767 & 0.58\end{array}$

$\begin{array}{lllllllll}\text { C5 } & -0.173 & -0.178 & -0.181 & -0.18 & -0.174 & -0.178 & -0.18 & -0.182\end{array}$

$\begin{array}{lllllllll}\text { C6 } & -0.182 & -0.187 & -0.19 & -0.19 & -0.179 & -0.185 & -0.188 & -0.19\end{array}$

$\begin{array}{lllllllll}\text { C7 } & -0.261 & -0.259 & -0.255 & -0.25 & -0.221 & -0.224 & -0.226 & -0.228\end{array}$

$\begin{array}{llllllllll}\text { C8 } & 0.3481 & 0.345 & 0.343 & 0.342 & 0.328 & 0.323 & 0.3198 & 0.318\end{array}$ 


\section{Conclusion}

1. In gas phase and solution at B3LYP/6-311++G (d,p) type DFT calculations yield the following results which estimates the stability of those tautomers is $\mathrm{X} 1>\mathrm{X} 2>\mathrm{X} 3$. $\mathrm{X} 1$ is the most stable form, but in gas phase, $(8-\mathrm{OH})$ subsisted is an exception that it has two forms. At the first one, hydrogen bond in hydroxyl substituent $(\mathrm{OH})$ with nitrogen is not formed and then the stability order is $\mathrm{X} 1>\mathrm{X} 2>\mathrm{X} 3$. The second form, the hydrogen bond is formed between them then the stability order is $\mathrm{X} 2>\mathrm{X} 1>\mathrm{X} 3$ and $\mathrm{X} 2$ isomer is dominant form. In all forms with increase of polarity, the total energy of all compounds is more negative.

2. The charges on all eight positions were affected by substituents and solvents.

3. The dipole moments of all compounds are affected by solvent. With increase of the polarity of solvents the dipole moments of the tautomers were increased.

\section{References}

1. Joule J A and Smith G F, Heterocyclic Chemistry, Reinhold V N, Ed., London, 1978, Second Ed., 1978, 82-83.

2. $\quad$ Li Q.-S and Fang W-H, Chem Phys Lett., 2003, 367(5-6), 637-644.

3. Mason S F, Philp J and Smith B E, J Am Chem Soc A, 1968, 3051.

4. Fang W-H, J Phys Chem A, 1999, 103(28), 5567-5573.

5. Tokay N and Ogretir C, J Mol Struct (Theochem), 2002, 594(3), 185-197.

6. Shchavlev A E, Pankratov A N and Shalabay A V, Int J Quantum Chem., 2006, 106(4), 876-886.

7. Zhao J-Y, Zhou Z-Y, Su Z-M, Xie Y-Z, Sun G-Y and Wu X, Chinese J Chem., 2006, 24(6), 724-730.

8. Miertus S, Scrocco E and Tomasi J, J Chem Phys., 1981, 55, 117.

9. Cances E, Mennucci V and Tomasi J, J Chem Phys., 1997, 107(8), 3032.

10. Cossi M, Barone V, Mennucci B and Tomasi J, Chem Phys Lett., 1998, 286(3-4), 253-260.

11. Barone V, Cossi M and Tomasi J, J Comp Chem., 1998, 19(4), 404-417.

12. Barone V and Cossi M, J Phys Chem A., 1998, 102(11), 1995-2001.

13. Reed A E, Curtiss L A and Wienhold F, Chem Rev., 1988, 88(6), 899-926.

14. Najafi Chermahini A, Nasr-Esfahani M, Dalirnasab Z, Abdol Dabbagh H and Teimouri A, J Mol Struct(Theochem)., 2007, 820(1-3), 7-11. 